\title{
Measurement and Simulation of Laser-Induced Fluorescence from Nonequilibrium Ultracold Neutral Plasmas
}

\author{
A. Denning \\ Brigham Young University \\ Scott D. Bergeson \\ Brigham Young University, scott.bergeson@byu.edu \\ F. Robicheaux \\ Auburn University
}

Follow this and additional works at: https://scholarsarchive.byu.edu/facpub

Part of the Physics Commons

\section{Original Publication Citation}

A. Denning, S. D. Bergeson, and F. Robicheaux. Measurement and simulation of laser-induced fluorescence from nonequilibrium ultracold neutral plasmas. Phys. Rev. A 80 (3), 033415 (2009).

\section{BYU ScholarsArchive Citation}

Denning, A.; Bergeson, Scott D.; and Robicheaux, F., "Measurement and Simulation of Laser-Induced Fluorescence from Nonequilibrium Ultracold Neutral Plasmas" (2009). Faculty Publications. 1833. https://scholarsarchive.byu.edu/facpub/1833 


\title{
Measurement and simulation of laser-induced fluorescence from nonequilibrium ultracold neutral plasmas
}

\author{
A. Denning, ${ }^{1}$ S. D. Bergeson, ${ }^{1}$ and F. Robicheaux ${ }^{2}$ \\ ${ }^{1}$ Department of Physics and Astronomy, Brigham Young University, Provo, Utah 84602, USA \\ ${ }^{2}$ Department of Physics, Auburn University, Auburn, Alabama 36849, USA
}

(Received 25 June 2009; published 25 September 2009)

\begin{abstract}
We report measurements and simulations of laser-induced fluorescence in ultracold neutral plasmas. We focus on the earliest times, when the plasma equilibrium is evolving and before the plasma expands. In the simulation, the ions interact via the Yukawa potential in a small cell with wrapped boundary conditions. We solve the optical Bloch equation for each ion in the simulation as a function of time. Both the simulation and experiment show the initial increase in ion fluorescence, disorder-induced heating, and coherent oscillation of the rms ion velocity. Detailed modeling of the fluorescence signal makes it possible to use fluorescence spectroscopy to probe ion dynamics in ultracold and strongly coupled plasmas.
\end{abstract}

DOI: 10.1103/PhysRevA.80.033415 PACS number(s): 32.50.+d, 52.20.-j, 52.25.Os, 52.65.Rr

\section{INTRODUCTION}

Ultracold neutral plasmas can be formed by photoionizing laser-cooled gases [1-6]. The initial ion temperature is approximately equal to the laser-cooled atom temperature, typically $0.01 \mathrm{~K}$ or less. These plasmas are also small. Their characteristic size is typically $1 \mathrm{~mm}$. The electron temperature can be tuned from approximately 0.5 to $1000 \mathrm{~K}$. At low initial electron temperature, space-charge effects prevent electrons from leaving, and the plasmas are charge neutral [1]. The electrons screen the ion-ion interactions, playing an important role in the rate at which the ions thermalize as the plasma evolves [5,7-30].

Immediately after photoionization, the ion-ion interaction raises the ion temperature. This happens because the ions are randomly distributed throughout the plasma. Although they have essentially zero kinetic energy, they have electrical potential energy. The ions move to minimize this potential energy, a process called disorder-induced heating [3,21]. The initial ion motion is coherent in the sense that all ions start moving simultaneously after photoionization, and damped oscillations have been observed in the ion velocity evolution.

During this initial plasma phase, the ion temperature is not well defined [21]. This is somewhat surprising because the initial nearest-neighbor distribution, which dominates the disorder induced heating phase, is approximately Gaussian. It has also been shown that the spatial distribution evolves self-similarly (i.e., is always Gaussian if the ion temperature is not too low) $[12,22]$. Furthermore, the velocity distributions both before and after the disorder-induced heating phase are Gaussian. However, the work of Ref. [21] showed that the velocity distribution during the disorder-induced heating phase is not Maxwellian. In particular, the higherorder velocity moment ratios $\frac{3}{5}\left\langle v^{4}\right\rangle /\left\langle v^{2}\right\rangle^{2}$ and $\frac{9}{35}\left\langle v^{6}\right\rangle /\left\langle v^{2}\right\rangle^{3}$ show substantial departures from Maxwellian values during this time.

Early fluorescence and absorption studies of ions in these plasmas used a Voigt profile to extract the effective ion temperature $[3,4]$. The Voigt profile,

$$
S(\nu)=\int L\left(\nu-x^{\prime}\right) G\left(x^{\prime}\right) d x^{\prime},
$$

is a convolution of the Lorentzian natural line shape $L(\nu)$ with the Gaussian $G(\nu)$ thermal line shape. While this appears to be valid in the later stages of the plasma evolution, the Gaussian width has limited meaning as the ions thermalize locally during the disorder-induced heating stage. Recent work suggests that velocity-changing collisions may broaden the Lorentzian profile in the plasma evolution [29].

Fluorescence measurements of ultracold neutral plasmas enable detailed studies of plasma dynamics. For strongly coupled neutral plasmas, departures from a collisionless Vlasov equation model have been observed [4,23]. One approach to studying the influence of strong coupling is to study these plasmas under ideal and weakly coupled conditions to ensure that the essential physics is understood, and then to move into the strongly coupled regime to look for variations from predicted behavior.

In this paper, we present a study of laser-induced fluorescence in ultracold neutral plasmas. Rather than extracting the temperature using a line-shape analysis or local fluorescence imaging, we perform a simulation of the fluorescence by numerically integrating the optical Bloch equations for the plasma ions. The simulated fluorescence signal is compared with experimental results. Both the experiment and simulation show the initial increase in fluorescence as the ions (all initially in the ground state) begin to absorb and scatter laser light. During the disorder-induced heating phase, the linewidth broadens and the fluorescence signal falls. At high densities, a coherent oscillation in the ion motion is observed. The experiment and simulation have satisfactory numerical agreement. We suggest ways to use these computational techniques in future experiments in strongly coupled neutral plasmas, particularly for extracting the effective ion and electron temperature at early times.

\section{EXPERIMENT}

Up to seven million ${ }^{40} \mathrm{Ca}$ atoms are laser cooled and trapped in a magneto-optical trap (MOT). The trap density is approximately Gaussian of the form 
(a) Ca I

(b) Ca II
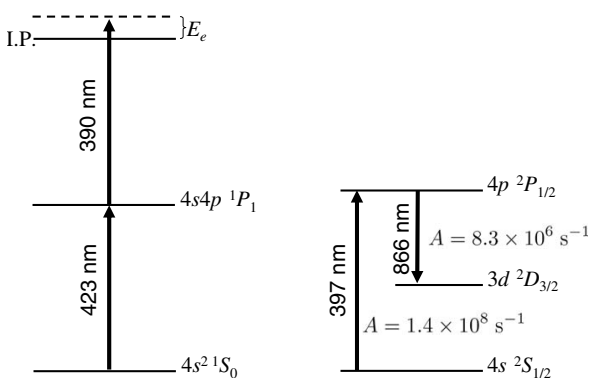

FIG. 1. Partial energy level diagram, transition wavelengths, and transition probabilities for relevant levels in (a) Ca I and (b) Ca II. The initial electron energy $E_{e}$ is determined by the energy difference between the photoionization laser photon energy and the $\mathrm{Ca}$ I ionization energy (IP). The electron temperature is $T_{e}=2 E_{e} / 3 k_{B}$. The $423 \mathrm{~nm}$ Ca I transition is also used for the calcium MOT. Not shown is a re-pumper laser at $672 \mathrm{~nm}$ that prevents an optical leak from the MOT.

$$
n(r)=n_{0} \exp \left(-r^{2} / 2 \sigma^{2}\right)
$$

where $n_{0}$ is the peak density with a typical value of $10^{10} \mathrm{~cm}^{-3}$ in our experiment, and $\sigma$ is the one-dimensional rms size of the MOT, typically $350 \mu \mathrm{m}$.

Atoms in the trap are photoionized using ns-duration pulsed lasers at 423 and $390 \mathrm{~nm}$. The temperature of the plasma ions is approximately equal to the $\sim \mathrm{mK}$ temperature of the MOT atoms. The initial energy of the plasma electrons is determined by the wavelength of the $390 \mathrm{~nm}$ laser. It is equal to the difference between the laser photon energy and the atomic ionization potential (see Fig. 1).

After the plasma is generated, plasma ions are excited using a cw laser beam tuned to the $4 s^{2} S_{1 / 2} \rightarrow 4 p{ }^{2} P_{1 / 2}$ transition at $397 \mathrm{~nm}$. Based on the in-loop feedback control signal, the frequency bandwidth of this laser is estimated to be less than $1 \mathrm{MHz}$. The $397 \mathrm{~nm}$ laser beam is focused to a Gaussian waist of $\sim 100 \mu \mathrm{m}$ in the center of the plasma. Fluorescence at this wavelength is collected using a lens, isolated using a band-pass optical interference filter, detected using a photomultiplier tube ( 2 ns full width at half maximum for single photon peaks), and recorded using a fast digital oscilloscope (500 $\mathrm{MHz}$ bandwidth). While most of the fluorescence from the upper ${ }^{2} P_{1 / 2}$ state is at $397 \mathrm{~nm}$, there is also a $6 \%$ branch to a dark metastable $3 d^{2} D_{3 / 2}$ state. Typical fluorescence curves are shown in Fig. 2.

The ions are all initially in the ground state. The fluorescence signal therefore rapidly rises at time $t=0$ as the ions scatter photons from the probe laser beam. As mentioned already, the ions initially accelerate due to the forces from neighboring ions in the plasma. This leads to disorderinduced heating, causing a rapid fall in the fluorescence signal after about 30 ns.

The plasma also expands because it is no longer confined by the MOT. When the initial electron energy is not too low, this plasma expansion velocity is approximated by the equation

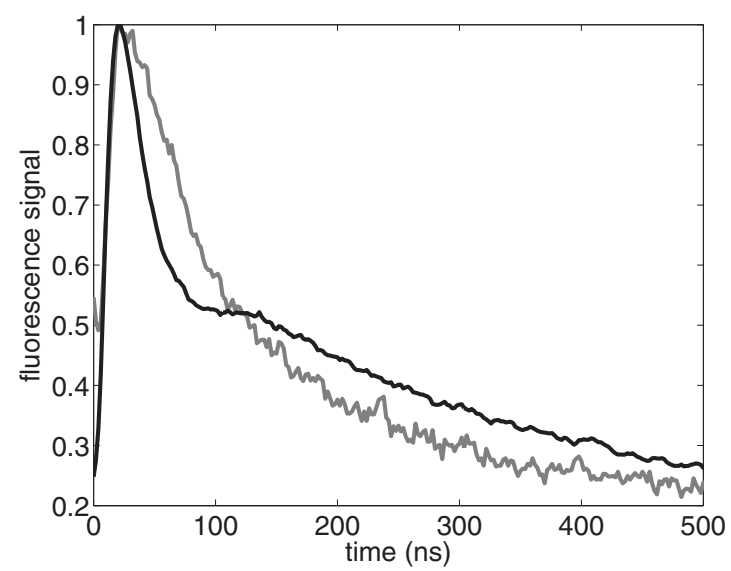

FIG. 2. Relative fluorescence signals from a high-density (black line, $n_{0}=7.3 \times 10^{9} \mathrm{~cm}^{-3}$ ) and low density (gray line, $n_{0}=0.45$ $\times 10^{9} \mathrm{~cm}^{-3}$ ) plasmas at $T_{e}=57 \mathrm{~K}$. In the high-density plasma, disorder induced heating occurs more rapidly. The fluorescence signal shows a weak oscillation around 100 ns. At 500 ns the signal has decayed somewhat less completely compared to the low density measurements. All fluorescence curves are in plotted in arbitrary units (linear scale) and have been normalized with a peak of 1 .

$$
v_{\exp }(r, t)=r \frac{2 k_{B} T_{e}}{m \sigma^{2}} t,
$$

where $r$ is the radial coordinate, $k_{B}$ is Boltzmann's constant, $T_{e}$ is the electron temperature, $m$ is the ion mass, and $t$ is time. This equation is valid for an isothermal electron model and can be used to calculate the plasma expansion velocity when the electron temperature is known. The electron temperature changes in time because of plasma expansion, energetic particle evaporation, electron-ion recombination, and electron-Rydberg atom collisions. For low temperature electrons, when the kinetic energy is comparable to the nearestneighbor Coulomb energy, this equation is not valid.

It is important to remember that Eq. (3) is a short-time approximation when the above conditions hold. A characteristic time for plasma expansion is when $v_{\exp }(\sigma, t)=\sigma / t$. For our conditions $\left(T_{e}=57 \mathrm{~K}\right.$ and $\left.\sigma=350 \mu \mathrm{m}\right)$ the characteristic time is approximately $2 \mu \mathrm{s}$. For times much shorter than this, Eq. (3) is appropriate. Our work focuses on the first 500 ns of plasma dynamics. During this time, the plasma ions accelerate. However, not enough time has passed for the plasma density to change. Similarly, changes in the electron temperature from recombination, evaporation, and scattering can be neglected based on estimates of these rates using our experimental conditions.

As can be seen in Fig. 2, the fluorescence method clearly distinguishes between high and low densities. Disorderinduced heating occurs more rapidly in high-density plasmas than in low-density plasmas. The fluorescence signal shows a weak oscillation around $100 \mathrm{~ns}$ in high-density plasmas that is absent in low-density plasmas. Finally, at $500 \mathrm{~ns}$, the fluorescence signal does not decay as completely for highdensity plasmas as it does for low density plasmas. For a fixed density, the fluorescence signal also changes as the initial electron temperature is reduced. If this signal can be properly interpreted, details about electron shielding, three- 
body recombination, many-body effects, and particle correlations can be determined at very early times in the plasma evolution.

\section{SIMULATION}

We simulate the plasma ion fluorescence signal by integrating the optical Bloch equations for a collection of ions in a cell. In the simulation, ions interact via the Yukawa potential,

$$
V(r)=\frac{q_{0}^{2}}{4 \pi \epsilon_{0}} \frac{e^{-r / \lambda_{D}}}{r},
$$

where $q_{0}$ is the fundamental charge, $\lambda_{D}=\sqrt{k_{B} T_{e} \epsilon_{0} / n q_{0}^{2}}$ is the Debye length, $k_{B}$ is Boltzmann's constant, $T_{e}$ is the electron temperature, $n$ is the density, and $\epsilon_{0}$ is the permittivity of free space.

The Yukawa model assumes that the electrons are in thermal equilibrium. The initial electron distribution is sharply peaked in energy and approaches a Maxwellian distribution during the first several ns, depending on density. We assume that the Yukawa potential is valid throughout the simulation. The present work can be seen as an indirect test of this assumption. We are developing a molecular dynamics model to explore the influence of the time-dependent electron distribution on Yukawa screening more carefully.

Plasma ions are randomly distributed over a cubic cell. The cell dimension is $L \approx \sigma / 10$. For the Gaussian density profile of Eq. (2), the density is constant across this cell size. We differentiate Eq. (4) to find the $x, y$, and $z$ components of the force on each ion due to the presence of all of the other (screened) ions in the cell. We use a fourth-order Runge Kutta stepper to move the ions in time.

At each time step, we also solve the optical Bloch equations for each ion in the cell. The ions are approximated as two-level atoms. The Hamiltonian for an ion is written in terms of the detuning $\omega$ and the Rabi frequency $\Omega$ as

$$
H=\hbar \omega \sigma_{-} \sigma_{+}+\frac{\hbar \Omega}{2}\left(\sigma_{-}+\sigma_{+}\right)
$$

where the raising and lowering matrices are

$$
\sigma_{+}=\left(\begin{array}{ll}
0 & 1 \\
0 & 0
\end{array}\right) \quad \sigma_{-}=\left(\begin{array}{ll}
0 & 0 \\
1 & 0
\end{array}\right) .
$$

The equation for the density matrix is

$$
\begin{aligned}
\dot{\rho}= & -i \omega\left(\sigma_{+} \sigma_{-} \rho-\rho \sigma_{+} \sigma_{-}\right)-i \frac{\Omega}{2}\left[\left(\sigma_{+}+\sigma_{-}\right) \rho-\rho\left(\sigma_{+}+\sigma_{-}\right)\right] \\
& +\frac{\gamma}{2}\left(2 \sigma_{-} \rho \sigma_{+}-\sigma_{+} \sigma_{-} \rho-\rho \sigma_{+} \sigma_{-}\right) .
\end{aligned}
$$

where $\gamma$ is the total decay rate of the $4 p^{2} P_{1 / 2}$ state. This equation of motion is somewhat more compactly written using the Pauli spin matrices, with $\sigma_{x}=\sigma_{+}+\sigma_{-}, \sigma_{y}=-i \sigma_{+}+i \sigma_{-}$, and $\sigma_{z}=\left(\begin{array}{cc}1 & 0 \\ 0 & -1\end{array}\right)$,

$$
\frac{d\left\langle\sigma_{z}\right\rangle}{d t}=\Omega\left\langle\sigma_{y}\right\rangle-\gamma\left(1+\left\langle\sigma_{z}\right\rangle\right),
$$

$$
\begin{gathered}
\frac{d\left\langle\sigma_{y}\right\rangle}{d t}=\omega\left\langle\sigma_{x}\right\rangle-\Omega\left\langle\sigma_{z}\right\rangle-\frac{\gamma}{2}\left\langle\sigma_{y}\right\rangle, \\
\frac{d\left\langle\sigma_{x}\right\rangle}{d t}=-\omega\left\langle\sigma_{y}\right\rangle-\frac{\gamma}{2}\left\langle\sigma_{x}\right\rangle .
\end{gathered}
$$

The fraction of atoms in the excited state is given by $\sigma_{z}$. For example, if there is no $397 \mathrm{~nm}$ laser, then $\Omega=0$ and the equation for $\sigma_{z}$ is

$$
\left\langle\sigma_{z}\right\rangle(t)=\left[1+\left\langle\sigma_{z}\right\rangle(0)\right] e^{-\gamma t}-1 .
$$

With the $397 \mathrm{~nm}$ laser present, $\Omega \neq 0$ and the fluorescence signal depends on time as

$$
f(t)=\frac{1}{2}\left[1+\left\langle\sigma_{z}(t)\right\rangle\right] .
$$

Because the Bloch equations depend on the detuning $\omega$ of the laser beam from the $397 \mathrm{~nm}$ resonance transition, we need to consider two frequency shifts. The first is the motion of the ions in the cell. This is given by the first-order Doppler shift,

$$
\Delta \omega=\frac{2 \pi \nu}{c} v,
$$

where $\nu$ is the atomic transition frequency in the rest frame of the atom, $c$ is the speed of light, and $v$ is the component of the atomic velocity along the direction of the laser beam propagation.

The second frequency shift comes from the radial acceleration of the plasma [Eq. (3)]. For early enough times in the plasma evolution, the ions accelerate and begin to move, but the overall density profile is unchanged. Therefore we can simulate the frequency shift due to plasma expansion by placing our cell at different plasma radii, calculating the mean velocity according to Eq. (3), and adding this directed velocity to all of the ion velocities in the cell.

For each time step, we calculate the position and velocity of each ion. We use wrapped boundary conditions to maintain a constant number of ions in the cell. We use the velocity (including the effect of plasma expansion) to determine the ion's frequency shift $\omega$ and use a second-order Runge-Kutta routine to calculate its $\sigma_{z}$. The value of $\sigma_{z}$ is then averaged over the ions in the cell to simulate the fluorescence signal. The simulation is performed for many different initial ion densities. The ion density is chosen randomly from a Gaussian distribution so that the different density regions are appropriately weighted.

Care must be taken to properly simulate the fluorescence decay into the optically dark $3 d$ state. At each time step the total fluorescence rate from the ${ }^{2} P_{1 / 2}$ level is calculated and multiplied by the branching ratio to get the decay rate into the dark $3 d^{2} D_{3 / 2}$ level. This decay rate is multiplied by the time step $d t$ to get the probability that the ion has made a transition to this level. As is traditionally done in Monte Carlo simulations, this probability is compared to a random number with a flat distribution between 0 and 1 . If the random number is less than the probability, then the simulated ion makes the transition to the dark state and no longer fluo- 


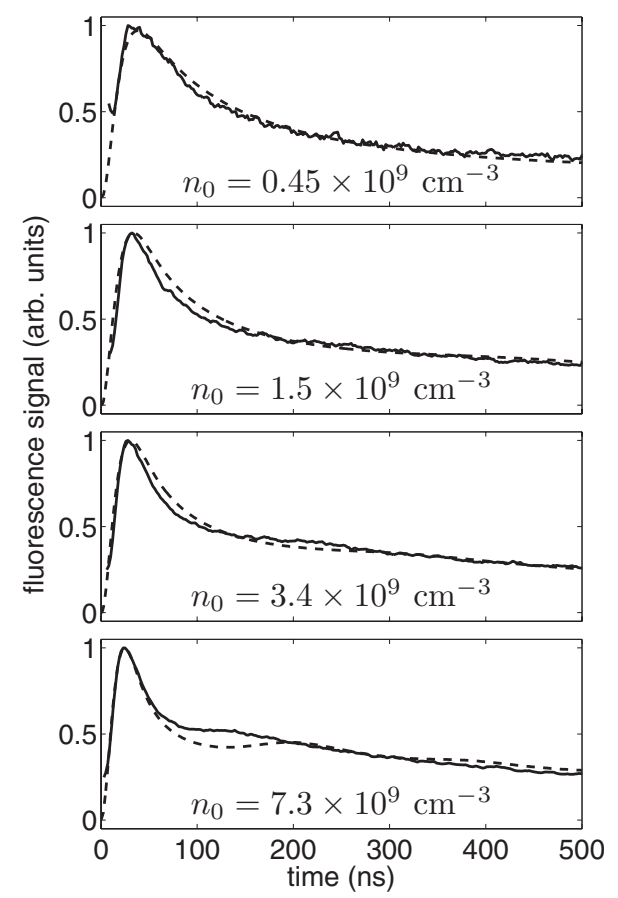

FIG. 3. Comparison of experiment (solid line) with simulation (dashed line) for a range of initial plasma densities. Both the simulation and experiment show initial onset of ion fluorescence, disorder-induced heating, and plasma expansion. As the density increases, the simulation shows the oscillation in the rms velocity distribution occurring later in time than in the experiment. This may be due to the onset of many-body interactions. For all of these measurements, the initial electron temperature is $T_{e}=57 \mathrm{~K}$.

resces. Decay into the dark metastable state reduces the fluorescence signal, particularly at longer times.

\section{COMPARISON WITH EXPERIMENT}

The simulation is compared with the experimental fluorescence signal in Fig. 3. Three phases of the signal are clearly visible. The first is the rapid rise of the fluorescence. This happens because the ions, which are all initially in the ground state, begin to scatter photons from the probe laser beam. The second is an almost equally rapid decline in fluorescence after $30 \mathrm{~ns}$. This is due to disorder-induced heating, the acceleration of the ions as they move to minimize their electrical potential energy. The third is the slower signal decay at longer times. This is due to the overall plasma expansion and to shelving into the $3 d$ state.

The agreement between the simulation and experiment is quite good. It suggests that the essential physics of the ion acceleration and plasma expansion are captured in this relatively simple approach.

There is an apparent discrepancy in the ion velocity oscillation at high density. When the plasma is generated, the ions begin to move in their local electrical potential energy well. After a time approximately equal to the inverse of the plasma frequency, the ions on average have moved to the other side of their local well and come to rest as they "collide" with their nearest neighbor. This motion-initially at rest, simultaneous acceleration, coming to rest again after one plasma period-produces an oscillation in the rms ion velocity distribution. Because nearest neighbors are randomly located in the initial plasma and because the plasma density is not uniform, the collision time is not exactly the same for all ions. After a short time, the coherence in the rms ion velocity oscillation is lost and the fluorescence curve smoothes out.

This discrepancy may be due to collisions or optical pumping in the plasma. Velocity-changing collisions and additional shifts from the MOT magnetic fields, and manybody interactions may also influence the fluorescence signal. These possibilities are currently under study.

Electron screening in ultracold neutral plasmas has been studied previously [14]. It was found that a Yukawa model reproduced the essential electron physics in weakly coupled plasmas. More recent work has confirmed this, with the caveat that evolving electron-temperature must be used in determining the screening length [23]. At the earliest times, during the first $100 \mathrm{~ns}$, the electron system is coming into equilibrium. Because three-body recombination and electron-Rydberg scattering both take time, the electron temperature in the earliest phase is determined primarily by disorder-induced heating $[9,25]$.

The work of [14] showed that electron screening changed the "final" temperature to which the plasma ions equilibrated at the end of their disorder-induced heating phase. This happens because screening softens the local potential in which the ions move. It was shown in [14] that the initial ion heating occurred during one plasma period. However, electron screening should also change the time scale for this heating. In fact, it may be possible to use the ion heating rate to estimate the electron screening length and thereby determine the electron temperature as it evolves during the earliest times in the plasma history.

\section{CONCLUSION}

We present measurements and simulations of laserinduced fluorescence of ions in an ultracold neutral plasma. In the simulation, the ions interact via the Yukawa potential. The ion position and velocity are calculated as a function of time, including the influence of ions in the cell as well as the overall plasma expansion. At each time step we solve the optical Bloch equations and track the excited-state population to simulate the fluorescence signal. We find good agreement between simulation and experiment.

Fluorescence measurements can be used to probe the early-time ion dynamics over a wide range of initial plasma densities. The ability to accurately calculate fluorescence properties of ultracold plasmas makes it possible to quantitatively interpret laser-induced fluorescence signals from ultracold plasmas. The influence of an external magnetic field, probe laser detuning, varying optical depth in the plasma, displacing the probe laser beam from the center of the plasma, and other important effects can be calculated as straightforward extensions of our approach.

Future work will focus on the agreement at higher densities in the plasma. This regime is important to understand because it is where many-body effects are likely to appear. Work at lower initial electron temperature is also interesting 
for the same reason. At the very lowest initial electron temperature or at the highest plasma densities, the energy scale in the simulation is determined only by the disorder-induced heating, both for electrons and ions. Comparing the Yukawa simulation, the experiment, and a direct molecular dynamics calculation would provide good insight into the onset of correlations in this regime.

\section{ACKNOWLEDGMENTS}

This work is supported in part by the Research Corporation, the National Science Foundation (Grant No. PHY0601699), and the Chemical Sciences, Geosciences, and Biosciences Division of the Office of Basic Energy Sciences, U.S. Department of Energy.
[1] T. C. Killian, S. Kulin, S. D. Bergeson, L. A. Orozco, C. Orzel, and S. L. Rolston, Phys. Rev. Lett. 83, 4776 (1999).

[2] S. Kulin, T. C. Killian, S. D. Bergeson, and S. L. Rolston, Phys. Rev. Lett. 85, 318 (2000).

[3] C. E. Simien, Y. C. Chen, P. Gupta, S. Laha, Y. N. Martinez, P. G. Mickelson, S. B. Nagel, and T. C. Killian, Phys. Rev. Lett. 92, 143001 (2004).

[4] E. A. Cummings, J. E. Daily, D. S. Durfee, and S. D. Bergeson, Phys. Rev. Lett. 95, 235001 (2005).

[5] R. S. Fletcher, X. L. Zhang, and S. L. Rolston, Phys. Rev. Lett. 96, 105003 (2006)

[6] T. C. Killian, Science 316, 705 (2007).

[7] T. C. Killian, M. J. Lim, S. Kulin, R. Dumke, S. D. Bergeson, and S. L. Rolston, Phys. Rev. Lett. 86, 3759 (2001).

[8] S. G. Kuzmin and T. M. O’Neil, Phys. Rev. Lett. 88, 065003 (2002).

[9] S. G. Kuzmin and T. M. O’Neil, Phys. Plasmas 9, 3743 (2002).

[10] S. Mazevet, L. A. Collins, and J. D. Kress, Phys. Rev. Lett. 88, 055001 (2002).

[11] F. Robicheaux and J. D. Hanson, Phys. Rev. Lett. 88, 055002 (2002).

[12] F. Robicheaux and J. D. Hanson, Phys. Plasmas 10, 2217 (2003).

[13] S. D. Bergeson and R. L. Spencer, Phys. Rev. E 67, 026414 (2003).

[14] Y. C. Chen, C. E. Simien, S. Laha, P. Gupta, Y. N. Martinez, P. G. Mickelson, S. B. Nagel, and T. C. Killian, Phys. Rev. Lett. 93, 265003 (2004).
[15] J. L. Roberts, C. D. Fertig, M. J. Lim, and S. L. Rolston, Phys. Rev. Lett. 92, 253003 (2004).

[16] W. Li et al., Phys. Rev. A 70, 042713 (2004).

[17] T. Pohl, T. Pattard, and J. M. Rost, Phys. Rev. A 70, 033416 (2004).

[18] T. Pohl, T. Pattard, and J. M. Rost, Phys. Rev. Lett. 94, 205003 (2005).

[19] E. A. Cummings, J. E. Daily, D. S. Durfee, and S. D. Bergeson, Phys. Plasmas 12, 123501 (2005).

[20] T. Pohl and T. Pattard, J. Phys. A 39, 4571 (2006).

[21] M. S. Murillo, Phys. Rev. Lett. 96, 165001 (2006).

[22] S. Laha, P. Gupta, C. E. Simien, H. Gao, J. Castro, T. Pohl, and T. C. Killian, Phys. Rev. Lett. 99, 155001 (2007).

[23] P. Gupta, S. Laha, C. E. Simien, H. Gao, J. Castro, T. C. Killian, and T. Pohl, Phys. Rev. Lett. 99, 075005 (2007).

[24] S. A. Ivanenko, E. A. Manykin, G. V. Naidis, B. B. Zelener, and B. V. Zelener, Laser Phys. 17, 419 (2007).

[25] S. D. Bergeson and F. Robicheaux, Phys. Rev. Lett. 101, 073202 (2008).

[26] S. L. Rolston, Physics 1, 2 (2008).

[27] X. L. Zhang, R. S. Fletcher, and S. L. Rolston, Phys. Rev. Lett. 101, 195002 (2008).

[28] X. L. Zhang, R. S. Fletcher, S. L. Rolston, P. N. Guzdar, and M. Swisdak, Phys. Rev. Lett. 100, 235002 (2008).

[29] J. Castro, H. Gao, and T. C. Killian, AIP Conf. Proc. No. 1114 (AIP, New York, 2009), p. 3.

[30] X. L. Zhang, R. S. Fletcher, and S. L. Rolston, AIP Conf. Proc. No. 1114 (AIP, New York, 2009), p. 11. 